\title{
Voltammetric determination of size and particle concentration of Cd-based quantum dots
}

\author{
Daniel Martín-Yerga ${ }^{a}$, Diego Bouzas-Ramos ${ }^{a}$, Mario Menéndez-Miranda ${ }^{\mathrm{b}}$, \\ Antonio R. Montoro Bustos ${ }^{\mathrm{b}}$, Jorge Ruiz Encinar ${ }^{\mathrm{b}}$, José M. Costa-Fernández ${ }^{\mathrm{b}}$, \\ Alfredo Sanz-Medel ${ }^{\mathrm{b}}$, Agustín Costa-García ${ }^{\mathrm{a}, *}$ \\ a Nanobioanalysis research group, Department of Physical and Analytical Chemistry, University of Oviedo, Spain \\ ${ }^{\mathrm{b}}$ Analytical spectrometry research group, Department of Physical and Analytical Chemistry, University of Oviedo, Spain
}

\section{A R T I C L E I N F O}

\section{Article history:}

Received 22 December 2014

Received in revised form 18 February 2015

Accepted 7 March 2015

Available online 11 March 2015

\section{Keywords:}

Quantum dots

Electrochemical characterization

Screen-printed electrodes

Voltammetry

Nanoparticles

\begin{abstract}
A B S T R A C T
In this article, we present two novel methodologies, using a simple electrochemical approach, for the determination of the size and particle concentration of Cd-based Quantum Dots (QDs), nanoparticles widely used as photoluminescent labels in many bioanalytical applications. Such QDs were analyzed directly in organic medium and in water after derivatization with an amphiphilic polymer. Screenprinted carbon electrodes modified with a bismuth film were employed as the electrochemical platform. The herein proposed methodologies allow the reliable determination of very low nanoparticle concentrations. Detection limits achieved with the selected experimental conditions were of $3.0 \times 10^{12}$ nanoparticles $\mathrm{mL}^{-1}$ for CdSe QDs dispersed in organic medium and of $6.0 \times 10^{12}$ nanoparticles $\mathrm{mL}^{-1}$ for water-solubilized CdSe/ZnS QDs (both with a core size of $3.26 \mathrm{~nm}$ ). However, detection limits could be improved increasing the QDs sample volume or the voltammetric deposition time. Furthermore, the proposed methodologies allowed the determination of the CdSe QDs diameters. Results obtained were validated after comparison with standard spectroscopic approaches.

The electrochemical characterization of QDs, disclosed in this work, allows to perform a synthesis control with a simple, inexpensive and fast approach.
\end{abstract}

(c) 2015 Elsevier Ltd. All rights reserved.

\section{Introduction}

Quantum dots (QDs) are semiconductor photoluminescent nanoparticles, with a roughly spherical shape and a nanoparticle diameter between 1-12 nm [1]. At such small sizes, the nanoparticles behave differently than the macroscopic material due, specially, to quantum confinement effects [2]. Some of their novel and improved characteristics are high photoluminescence emission quantum yields, size-tunable emission profiles and narrow spectral bands [3]. Nowadays it is well recognized that the special optoelectronic properties exhibited by the QDs, as well as the toxicological and physicochemical behavior of these type of nanocrystals, are strongly affected by the size, nanoparticle concentration and nature of the nanoparticles.

\footnotetext{
* Corresponding author at: Nanobioanalysis group, Department of Physical and Analytical Chemistry, University of Oviedo, 8 Julián Clavería St., Oviedo 33006 Spain. Tel.: +34 985103488; fax: +34 985103125 .

E-mail address: costa@uniovi.es (A. Costa-García).
}

Actually, the size-dependent properties of QDs make the characterization of these nanomaterials a mandatory postsynthesis stage before their use as labels in bioanalytical applications. QDs physico-chemical characteristics have been extensively studied by different techniques such as inductively coupled plasma mass spectrometry (ICP-MS), photoluminiscence, X-ray photoelectron spectroscopy (XPS), X-ray diffraction or electron microscopy [4-7]. These techniques have several advantages and disadvantages, but generally speaking it can be said that their use can be laborious, time consuming or requiring expensive instrumentation. Electrochemical techniques have been also used for the characterization of QDs, providing valuable information about the nanocrystals, such as energy bands [8] or surface defects [9]. For instance, cyclic voltammetry has been used to study the quantum confinement and band structure parameters in oleic acid stabilized CdTe dispersions, as a function of nanoparticle size [10]. Bard's group have extensively studied other electronic and electrochemical properties of QDs using voltammetric techniques [11,12]. Recently, Amelia et al. [13] and Huang et al. [14] have evaluated the published literature about the electrochemical properties and applications of these semiconductor nanoparticles, 
respectively. Voltammetric measurements have been previously used for the quantification and stability studies of quantum dots $[15,16]$. For instance, Hynek et al. [17] have measured the electrochemical changes of aqueous solutions of QDs such as PbS, $\mathrm{CdS}, \mathrm{CdTe}$ and CuS modified with different capping agents using several aging and storage conditions. They have been also used as detectors in biosensing applications based on the use of QDs as voltammetric [18,19] or electrochemiluminescence [20] labels. However, a direct and unequivocal relationship between the QDs size and their electrochemical properties has not been described yet. Therefore, the development of a simple and rapid method for the routine determination of QDs size and concentration using fast and simple electrochemical techniques may result highly valuable.

In this context, the main objective of this work was to develop a fast, inexpensive and easy method for the sensitive determination of the concentration and size of Cd-based QDs. This electrochemical characterization is based on the measurement of $\mathrm{Cd}(\mathrm{II})$ released from the QDs, after a nanoparticle acid attack. Screen-printed electrodes modified with a bismuth film were employed as the electrochemical platform due to its small size, low cost and disposable nature.

\section{Materials}

\subsection{Apparatus and electrodes}

UV-Vis absorption spectra were recorded at room temperature on a Genesys $10 \mathrm{~S}$ Thermo Scientific Spectrophotometer (Thermo Scientific, Germany). Fluorescence spectra were recorded on a Varian Cary Eclipse (Agilent, Germany) fluorescence spectrophotometer using a fixed excitation wavelength of $350 \mathrm{~nm}$ with excitation and emission slit widths of $10 \mathrm{~nm}$. All measurements were carried out using fluorescence-type quartz cuvettes.

Voltammetric measurements were performed with an Autolab PGSTAT 12 (Eco Chemie, The Netherlands) potentiostat and controlled by Autolab GPES 4.9. All measurements were carried out at room temperature. Screen-printed carbon electrodes (SPCEs, Ref. 110) were purchased from DropSens (Spain).

\subsection{Reagents and solutions}

All chemicals used for QD synthesis and surface modification were of analytical grade and used as received without further purification. The precursors used for the synthesis of the metal nanoparticles were selenium powder (99.99\%), cadmium oxide (99.99\%), trioctylphosphine (TOP, 90\%), trioctylphosphine oxide (TOPO, 99\%) and anhydrous chloroform ( $\geq 99 \%$ ), all of them purchased from Sigma Aldrich (Schnelldorf, Germany). Hexylphosphonic acid (HPA) was obtained from Alfa Aesar (Karlsruhe, Germany). Cadmium(II) acetate, sodium hydroxide, sulfuric acid (97\%), acetic acid (100\%) nitric acid (65\%), perchloric acid (70\%) and hydrochloric acid (37\%) were purchased from Merck. Bismuth(III) standard, Tris(hydroxymethyl) aminomethane (Tris) and CdSe QDs (Lumidot CdSe) were purchased from Sigma-Aldrich. Ultrapure water obtained with a Millipore Direct $Q 5^{\mathrm{TM}}$ purification system from Millipore Ibérica S.A. (Madrid, Spain) was used throughout this work. All other reagents were of analytical grade. Working solutions of CdSe QDs were made by appropriate dilution of the QDs batch solution in $\mathrm{CHCl}_{3}$. Working solutions of $\mathrm{CdSe} / \mathrm{ZnS}$ QDs surfacemodified for water solubility were made by appropriate dilutions of the concentrated batch solution in $0.1 \mathrm{M} \mathrm{pH} \mathrm{7.4} \mathrm{Tris-HCl} \mathrm{buffer.}$

\subsection{CdSe and CdSe/ZnS Quantum dots synthesis}

CdSe QDs were synthesized using CdO as precursor via the organometallic route described by Peng's group [21] with slight modifications. Briefly, $1.23 \mathrm{mmol}$ of CdO, $0.6 \mathrm{~g}$ of HPA and $17.5 \mathrm{~g}$ of TOPO were loaded into a $250 \mathrm{~mL}$ glass three-necked flask. The mixture was heated up to $300-320^{\circ} \mathrm{C}$ under argon flow for 15-20 min to allow the complete dissolution of CdO in HPA and TOPO. After cooling the temperature of the solution down to $270^{\circ} \mathrm{C}, 6 \mathrm{~mL}$ of a selenium stock solution prepared in an Ar-filled dry-box by dissolving $1.63 \mathrm{mmol}$ of selenium powder in $7.5 \mathrm{~mL}$ of TOP was swiftly injected. After injection, nanocrystals were left to grow from 5 to $15 \mathrm{~min}$ at $250^{\circ} \mathrm{C}$ depending on the desired core size and for hence fluorescence emission wavelength desired.

In the case of the "core-shell"-type CdSe/ZnS QDs, the CdSe core of the QDs was synthesized following the approach previously described. Once the growth process has reached the desirable CdSe core size, $7.5 \mathrm{~mL}$ of a zinc sulphide (Zn/S/TOP) stock solution (prepared in Ar atmosphere by mixing $1.18 \mathrm{mmol}$ of hexamethyldisilathiane, $8.34 \mathrm{mmol}$ of diethyl zinc and $10.25 \mathrm{~mL}$ of TOP) was added slowly at $230^{\circ} \mathrm{C}$ in order to allow the generation of the $\mathrm{ZnS}$ shell, and then it was cooled down to $100^{\circ} \mathrm{C}$ for an hour. In order to remove excess of unreacted QDs precursors and reagents the reaction mixture was then centrifuged to precipitate the QDs dispersed. Then the supernatant was discarded and the precipitated QDs were redispersed in anhydrous chloroform and stored at room temperature in the dark before use.

\subsection{Water solubilization of $Q D s$}

In order to make the nanoparticles hydrophilic to render them compatible with aqueous media, the QDs were surface-modified with an amphiphilic polymer (based on poly-maleic anhydride functional groups), following a procedure described elsewhere [22].

\subsection{Spectroscopic characterization}

The quality of the synthesized QDs was first evaluated by registering their VIS-UV absorbance and fluorescence spectra. Absorption spectrophotometric measurements can be used to estimate the size, concentration and extinction coefficient of the QDs, following the approach proposed by Peng and coworkers [23]. Additionally, the full width at half maximum (fwhm) of the fluorescence emission is a commonly employed parameter to estimate the size distribution of the nanoparticles (fwhm values lower than $35-40 \mathrm{~nm}$ is considered to indicate low QDs sizedispersion).

Four different CdSe QDs synthesis were carried out varying reaction times and precursors concentration in order to obtain QDs with different nanoparticle size. The core size, the particle concentration (both calculated according to Peng،s equations) and the maximum emission wavelength of the CdSe QDs obtained after the different syntheses are collected in Table 1. Fig. 1A shows the fluorescence emission spectra for the different organic CdSe QDs syntheses. As can be seen, all organic CdSe QDs synthesis showed an intense fluorescence emission band and a fwhm of around $30 \mathrm{~nm}$, indicating a very low dispersion of the QDs diameter sizes.

Table 1

Diameter, concentration and wavelength emission maximum of the synthesized CdSe QDs and CdSe/ZnS QDs.

\begin{tabular}{llll}
\hline QDs & Diameter $(\mathrm{nm})$ & $\lambda_{\text {em.max. }}(\mathrm{nm})$ & Concentration $(\mu \mathrm{M})$ \\
\hline CdSe synthesis 1 & 2.77 & 555 & 3.75 \\
CdSe synthesis 2 & 2.98 & 575 & 2.65 \\
CdSe synthesis 3 & 3.14 & 580 & 5.51 \\
CdSe synthesis 4 & 3.26 & 590 & 4.11 \\
CdSe/ZnS & 3.26 & 590 & 1.61 \\
\hline
\end{tabular}


A)

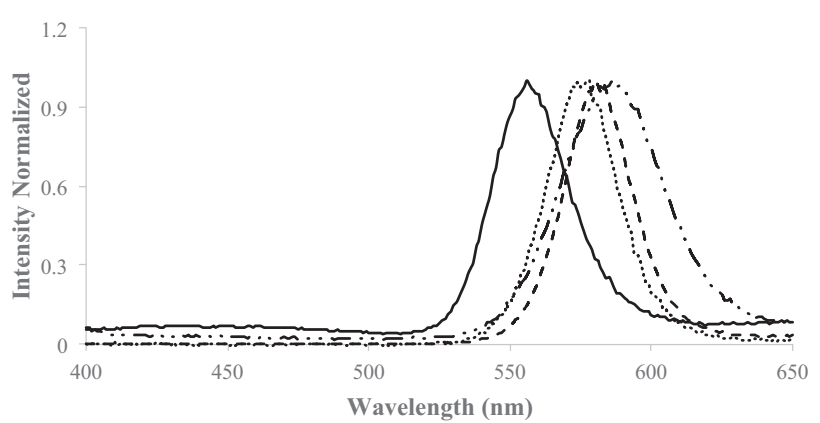

B)

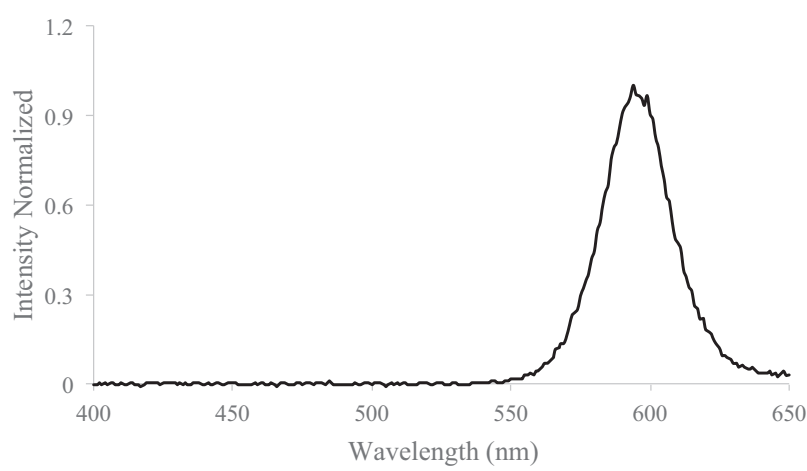

Fig. 1. (A) Fluorescence emission spectra acquired in $\mathrm{CHCl}_{3}$ for the different $\mathrm{CdSe}$ QDs syntheses under study (see Table 1). Synthesis 1, Solid Line; Synthesis 2, Dotted line; Synthesis 3, Dashed line; Synthesis 4, Dashed-Dot line. (B) Fluorescence emission spectrum of CdSe/ZnS QDs in water media.

Fluorescence spectrum for a new synthesis of the watersolubilized CdSe/ZnS QDs is plotted in Fig. 1B. Again, the narrow emission spectra obtained indicates the low size-distribution of the NPs obtained. The core size, the particle concentration (both calculated according to Peng's equations) and the maximum emission wavelenght are also described in Table 1.

\section{Methods}

\subsection{Dissolution of QDs and voltammetric measurement}

\subsubsection{CdSe QDs in organic medium}

An acid attack of the QDs is necessary to release the cadmium ions that are then electrochemically measured. Typically, $10 \mu \mathrm{L}$ of QDs in $\mathrm{CHCl}_{3}$ were placed in an eppendorf tube and after total evaporation of $\mathrm{CHCl}_{3}, 10 \mu \mathrm{L}$ of $\mathrm{HClO}_{4} 1 \mathrm{M}$ was dropped for the dissolution of the QDs. Previously, it has been reported in the literature that a concentrated acid is able to etch and dissolve Cd-based quantum dots [24,25]. Therefore, we could assume that the attack of CdSe QDs with concentrated acid is likely to follow a similar reaction to macroscopic CdSe (Eq. (1)).

$\mathrm{CdSe}+2 \mathrm{HClO}_{4} \rightarrow \mathrm{Cd}^{2+}+2 \mathrm{ClO}_{4}^{-}+\mathrm{H}_{2} \mathrm{Se}$

After gently shaking to ensure an efficient QDs acid-attack, $10 \mu \mathrm{L}$ of $\mathrm{NaOH} 1 \mathrm{M}$ was added to neutralize the excess of acid. Then, $80 \mu \mathrm{L}$ of $0.1 \mathrm{M}$ pH 5.0 acetate buffer, containing $1.25 \mu \mathrm{g} \mathrm{mL}^{-1}$ of Bi (III), was added and mixed with the previous solution. An aliquot of $40 \mu \mathrm{L}$ of the resulting solution was added to the electrode. A constant potential of $+0.40 \mathrm{~V}$ was applied for $60 \mathrm{~s}$ to activate the working electrode and a deposition potential of $-1.30 \mathrm{~V}$ for $120 \mathrm{~s}$ (200 s for concentration measurements) was applied producing the reduction of $\mathrm{Cd}(\mathrm{II})$ (Eq. (2)) and $\mathrm{Cd}(0)$ is preconcentrated on the electrode surface.

$\mathrm{Cd}(\mathrm{II})+2 \mathrm{e}^{-} \rightarrow \mathrm{Cd}(0)$

Then, the potential was swept from $-1.30 \mathrm{~V}$ to $-0.80 \mathrm{~V}$ using differential pulse voltammetry (DPV) with optimized parameters, and the reoxidation (stripping) of $\mathrm{Cd}(0)$ to $\mathrm{Cd}(\mathrm{II})$ was carried out, generating a current intensity (Eq. (3)). An scheme of the procedure is shown in Fig. $2 \mathrm{~A}$.

$\mathrm{Cd}(0) \rightarrow \mathrm{Cd}(\mathrm{II})+2 \mathrm{e}^{-}$

\subsubsection{CdSe/ZnS QDs in aqueous medium}

Typically, $10 \mu \mathrm{L}$ of aqueous QDs solution was dropped in the working electrode of a SPCE. Next, $1 \mu \mathrm{L}$ of $\mathrm{HCl} 1 \mathrm{M}$ was added to the electrode for the dissolution of QDs. Similarly to the CdSe case, for CdSe/ZnS QDs is expected that the acid attack result in the reactions described in Eq. (4) and Eq. (5):

$\mathrm{CdSe}+2 \mathrm{HCl} \rightarrow \mathrm{Cd}^{2+}+2 \mathrm{Cl}^{-}+\mathrm{H}_{2} \mathrm{Se}$

$\mathrm{ZnS}+2 \mathrm{HCl} \rightarrow \mathrm{Zn}^{2+}+2 \mathrm{Cl}^{-}+\mathrm{H}_{2} \mathrm{~S}$

Then, $30 \mu \mathrm{L}$ of $0.1 \mathrm{M}$ pH 4.5 acetate buffer, containing $1.3 \mu \mathrm{g}$ $\mathrm{mL}^{-1}$ of $\mathrm{Bi}(\mathrm{III})$, was added over the previous solution. A constant potential of $+0.40 \mathrm{~V}$ was applied for $60 \mathrm{~s}$ to activate the working electrode and a deposition potential of $-1.10 \mathrm{~V}$ for $200 \mathrm{~s}$ was applied. Identical redox reactions occur in this case, as described in Eqs. (2) and (3). The potential was swept from $-1.10 \mathrm{~V}$ to $-0.80 \mathrm{~V}$ using differential pulse voltammetry (DPV) with the optimized parameters. A scheme of the procedure is shown in Fig. $2 \mathrm{~B}$.

A)

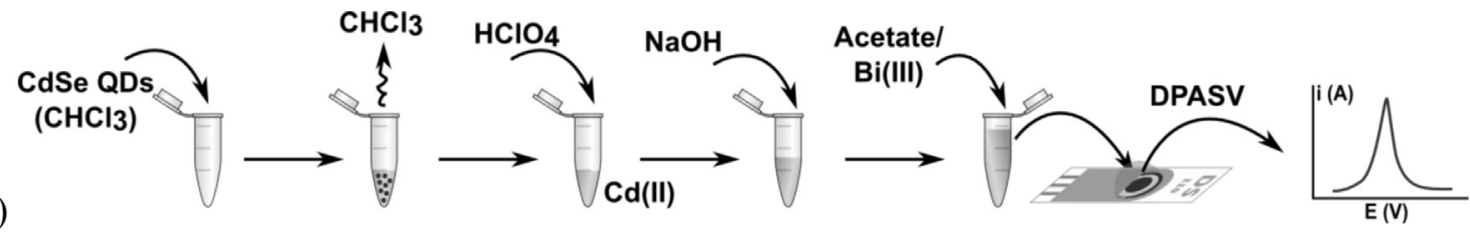

B)
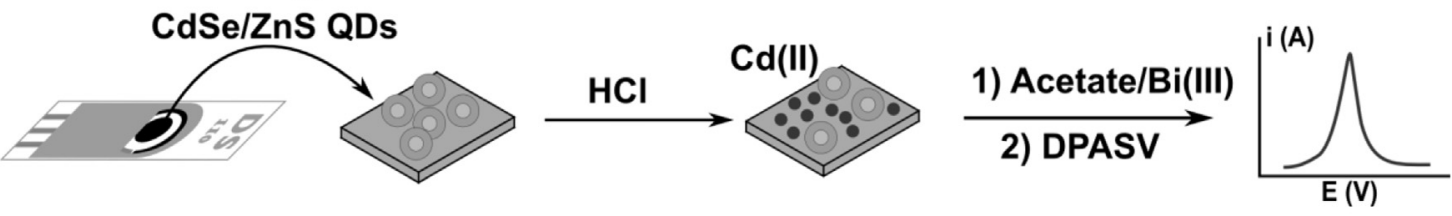

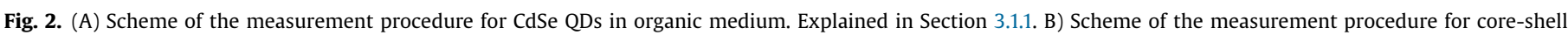
$\mathrm{CdSe} / \mathrm{ZnS}$ QDs in aqueous medium. Explained in Section 3.1.2. 
3.2. Optimization of the Bi-modified screen-printed electrodes for Cd (II) detection

For the electrochemical characterization of Cd-based QDs is essential to choose a suitable electrochemical platform for $\mathrm{Cd}(\mathrm{II})$ detection. Screen-printed electrodes modified with a bismuth film were chosen as an excellent platform for the detection of $\mathrm{Cd}(\mathrm{II})$ and Cd-based QDs, as reported in the literature [26,27]. The reference technique in such works is the highly sensitive and simple anodic stripping voltammetry. Cadmium deposition on the electrode is performed on a bismuth film, enhancing the preconcentration. The optimization of the Bi film is critical to achieve a sensitive method. Therefore, the assessment of several conditions influencing the bismuth film was carried out.

Initially, the generation of the Bi film by electrodeposition on SPCE with in situ and ex situ approaches, already known in the literature $[28,29]$, was studied. In a previous work, an improvement of the magnitude and reproducibility of the voltammetric signal of $\mathrm{Cd}(\mathrm{II})$ using these electrodes was observed after the activation of the electrode surface by applying a positive potential
[30]. In this case, a potential of $+0.4 \mathrm{~V}$ for $60 \mathrm{~s}$ was chosen as the activation step. Several concentrations of $\mathrm{Bi}(\mathrm{III})$, deposition potentials and deposition times were considered to obtain the best electrodic surface for the detection of $\mathrm{Cd}(\mathrm{II})$ using anodic stripping differential-pulse voltammetry (ASDPV) (data not shown). In situ generation of the bismuth film, using $1 \mu \mathrm{g} \mathrm{mL} L^{-1}$ of $\mathrm{Bi}(\mathrm{III})$, was finally chosen as the best suitable methodology for the sensitive and reproducible analysis of cadmium.

\subsection{Optimization of the experimental conditions}

\subsubsection{CdSe QDs in organic medium}

In the here-proposed approach, it is necessary an acid digestion of the QDs to release $\mathrm{Cd}(\mathrm{II})$ into the solution prior to the electrochemical metal detection. By this way, the sensitivity is greatly enhanced compared to the direct detection of QDs and further elemental information on the nanoparticle could be obtained.

Initially, a simple digestion step carried out directly on the electrode surface after the deposition of few $\mu \mathrm{L}$ QDs (dispersed in
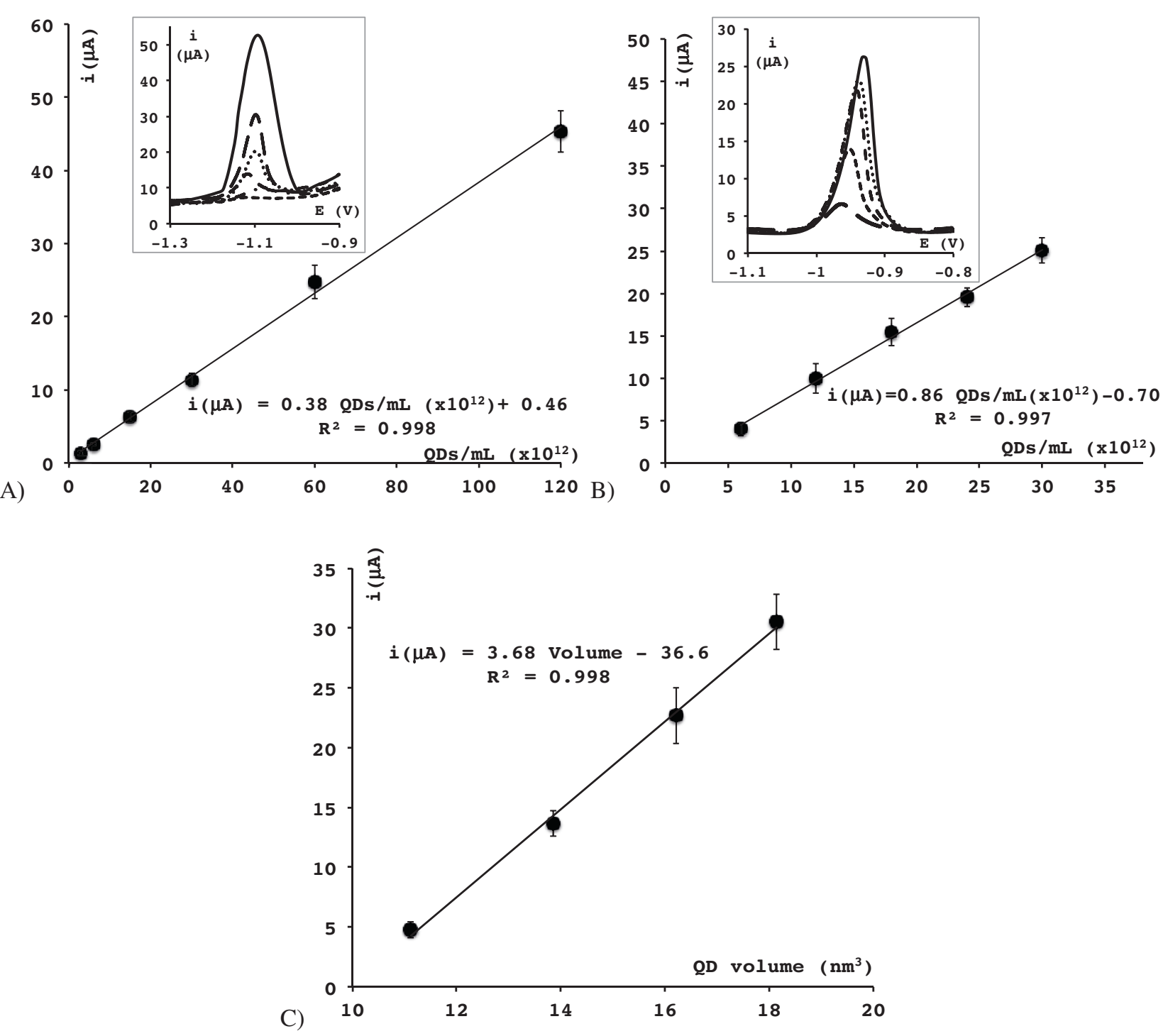

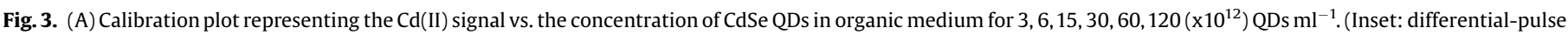

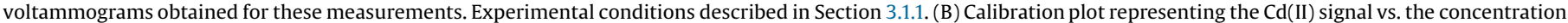

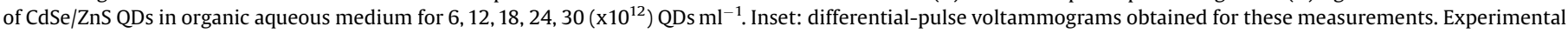
conditions described in Section 3.1.2. (C) Relation plot between Cd(II) signal vs. the volume for CdSe QDs of 2.77, 2.98, 3.14 and 3.26 nm. 
$\mathrm{CHCl}_{3}$ ) was carried out. However, the $\mathrm{CHCl}_{3}$ damaged the electrode ink and the analytical results were not reproducible. Therefore, an alternative methodology for the QDs digestion, consisting in the nanoparticle acid attack outside of the electrode was carried out. The protocol followed was detailed in Section 3.1.1. The QDs sample volume chosen was $10 \mu \mathrm{L}$ with a concentration of $100 \mathrm{nM}$ because it was found to be high enough to get a very sensitive analytical signal in the working range. It is worth noting that if higher sensitivity is needed, the volume of the QDs solution may be increased so a bigger amount of nanoparticles would be available for the acid attack, after evaporation of the $\mathrm{CHCl}_{3}$.

Several acids $\left(\mathrm{H}_{2} \mathrm{SO}_{4}, \mathrm{HNO}_{3}, \mathrm{HCl}\right.$ and $\left.\mathrm{HClO}_{4}\right)$ and different experimental conditions were used for the optimization of QDs dissolution. The best results in terms of signal/noise ratio were obtained with $1 \mathrm{M} \mathrm{HClO}_{4}$ and a volume of $10 \mu \mathrm{L}$ (assuring that all the nanoparticles were in contact with the acid). To neutralize the excess of acid, $10 \mu \mathrm{L}$ of $\mathrm{NaOH} 1 \mathrm{M}$ was employed. This neutralization was necessary because a more acidic medium could interfere with the electrochemical detection of $\mathrm{Cd}(\mathrm{II})$, since the $\mathrm{H}^{+}$reduction occurs at a close potential. Acetate buffer $0.1 \mathrm{M} \mathrm{pH} 5.0$ (optimized conditions) was used for the measurement because it is the most used electrolytic medium for the determination of heavy metals even after their release from QDs employed as biosensing label [31]. Several parameters related to the anodic stripping differential-pulse voltammetry were optimized: deposition potential $(-1.3 \mathrm{~V})$, amplitude $(0.1 \mathrm{~V})$, step $(6 \mathrm{mV})$, modulation time $(8 \mathrm{~ms})$ and interval time $(1.5 \mathrm{~s})$. The deposition time was chosen as a function of the latter application. In this sense, a longer deposition time of $200 \mathrm{~s}$ was chosen for the determination of QDs concentration, where a more sensitive method was preferred, whereas for size characterization studies, $120 \mathrm{~s}$ was enough.

\subsubsection{CdSe/ZnS QDs in aqueous medium}

For the electrochemical characterization of the core-shell CdSe/ZnS QDs in aqueous medium, several parameters were optimized such as the sample volume, the nature, volume and concentration of the acid used for the nanoparticle digestion and the detection buffer. The optimized values are listed in the Section 3.1.2. It is worth mentioning that QDs dissolution may not be complete, since the relation between the sample and acid volume is $10: 1$. However, this is not affecting final quantitative results. In fact, the use of a higher volume or acid concentration leads to less sensitive detection since the excess of $\mathrm{H}^{+}$results in $\mathrm{H}_{2}$ generation at the electrode surface, decreasing the deposition efficiency of the released cadmium.

\section{Applications}

\subsection{Determination of QDs Concentration}

\subsubsection{CdSe QDs in organic medium}

The amount of $\mathrm{Cd}(\mathrm{II})$ released after the acid attack is directly proportional to the amount of QDs in solution, as it has been previously reported [32]. In order to prepare a calibration plot, a solution of $6.0 \times 10^{14} \mathrm{QDs} \mathrm{mL}^{-1}(1.0 \mu \mathrm{M})$ with a nanoparticle size of $3.26 \mathrm{~nm}$ (determined spectroscopically), was diluted in $\mathrm{CHCl}_{3}$ in order to prepare a set of working solutions with different nanoparticle concentrations. These diluted QDs organic solutions were then analysed following the general procedure. As shown in Fig. 3A, the developed method resulted in a calibration plot with high linearity in a range between $3.0 \times 10^{12}-1.2 \times 10^{14} \mathrm{QDs} \mathrm{mL}^{-1}$

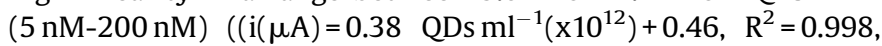
$\mathrm{RSD}=5.6 \%, \mathrm{n}=3)$ and a calculated detection limit of $1.6 \times 10^{12} \mathrm{QDs} \mathrm{mL}^{-1}(2.6 \mathrm{nM})$. Considering the sample volume analysed $(10 \mu \mathrm{L})$, the detection limit corresponds to just $1.6 \times 10^{10}$ nanoparticles (27 fmol of QDs).
Data obtained show a high relationship between the concentration of CdSe QDs in solution and the voltammetric signal measured from the $\mathrm{Cd}^{2+}$ released after acid attack. Therefore, this method can be used satisfactorily for the determination of QDs in organic solution. Although the limit of detection achieved is significantly higher than that of the ICP-MS (18 pM), the electrochemical determination of QDs could be still useful for a wide variety of applications due to the simplicity of the procedure and lower cost (in terms of both investment and running cost) of the instrumentation employed.

\subsubsection{CdSe/ZnS QDs in aqueous medium}

Likewise, the concentration of core-shell CdSe/ZnS QDs in aqueous medium might be correlated to the voltammetric signal obtained after the acid attack. Using the optimized methodology described in Section 3.1.2, a calibration plot was carried out (Fig. 3B). A linear range between $6.0 \times 10^{12} \mathrm{QDs} \mathrm{mL}^{-1}(10 \mathrm{nM})$ and $3.0 \times 10^{13} \mathrm{QDs} \mathrm{mL}^{-1}(50 \mathrm{nM})$ was obtained $(\mathrm{i}(\mu \mathrm{A})=0.86$ QDs $\left.\mathrm{ml}^{-1}\left(\mathrm{x} 10^{12}\right)-0.70, \mathrm{R}^{2}=0.997, \mathrm{RSD}=8.8 \%, \mathrm{n}=3\right)$ and a calculated detection limit of $2.0 \times 10^{12} \mathrm{QDs} \mathrm{mL}^{-1}(3.3 \mathrm{nM})$. This linear range, smaller in comparison to the methodology for CdSe QDs in organic medium, may be due to several reasons such as the difference between the acid and sample volume and a less effective acid attack due to the more complex surface coating (water-soluble QDs are coated with a ZnS shell and an amphiphilic polymer, based on poly-maleic anhydride functional groups). However, this methodology is much simpler and also allows the determination of very low concentrations of core-shell QDs in aqueous medium, $2.0 \times 10^{12} \mathrm{QDs} \mathrm{mL}^{-1}(3.3 \mathrm{nM})$, that considering the sample volume analysed $(10 \mu \mathrm{L})$, it corresponds to the detection of just $2.0 \times 10^{10}$ QDs (37 fmol of QDs). Compared to previous work carried out by Merkoçi et al. [15] $\left(1 \times 10^{14}\right.$ QDs, 0.15 nanomoles of QDs), an improvement of more than three orders of magnitude has been achieved.

\subsection{Determination of QDs Size}

The eventual surface-modifications of the QDs, typically used to solubilize them, increase their stability by minimizing aggregations or provide them with functional activity, can increase their actual total nanocrystal size, and affect their physicochemical properties. However, only the diameter (size) of the core of the nanoparticle affects their optoelectronic properties. However, for core-shell QDs, many often uncontrolled mixed multilayers containing Cd may appear over the nanoparticle core. That means that a variable amount of total $\mathrm{Cd}$ can be found in the modified particle, which does not allow establishing a direct relationship between the total amount of $\mathrm{Cd}$ and the sought parameter, the size of the core. For this reason, the characterization of QDs sizes was carried out using unmodified CdSe QDs in organic medium, since all the $\mathrm{Cd}$ measured could be related to their core and therefore, to their optoelectronic properties.

Organic working solutions containing QDs of several sizes, previously characterized by spectroscopic techniques $(2.77,2.98$, 3.14 and $3.26 \mathrm{~nm}$ ), were used for validation of the electrochemical methodology. The core size of the CdSe QDs may be correlated to the voltammetric signal obtained after the acid attack. Using the same optimized experimental procedure already employed in the experiments described in Section 4.1.1, the characteristic electrochemical signal for $\mathrm{Cd}(\mathrm{II})$ was obtained. As expected, significant differences were observed between the $\mathrm{Cd}$ signals obtained for each size. Since the cadmium signal is related to the QDs concentration and size, we are not able to determinate simultaneously both, so being necessary to know the number of QDs before the size can be determined. Therefore, it should be mentioned that the concentration of QDs measured was always 
the same for all QDs sizes. In this case, $6.0 \times 10^{13} \mathrm{QDs} \mathrm{mL}^{-1}(100 \mathrm{nM}$ of QDs) was chosen. Fig. 3C shows the high linear relationship between the volume of the nanoparticle (assuming a spherical nanoparticle shape with a volume of $\left.4 / 3 \pi r^{3}\right)$ and the electrochemical signal measured $\left(i(\mu \mathrm{A})=3.68 \mathrm{~V}\left(\mathrm{~nm}^{3}\right)-36.6, \mathrm{R}^{2}=0.998\right.$, $\mathrm{RSD}=9.1 \%, \mathrm{n}=6$ ).

An important advantage of the herein proposed methodology is the versatility to determine different QDs sizes outside of the sizerange tested in this work. The concentration of QDs may be changed towards higher values if it is necessary to measure smaller sizes, what provides enough $\mathrm{Cd}$ atoms to be detected. Conversely, QDs concentration may be changed towards lower values if the QD size is higher in order to avoid Cd signal saturation.

The developed method was finally tested for the characterization of CdSe QDs synthesized using alternative synthetic routes different. For such purpose, a solution of commercial CdSe QDs (core-type without shell) diluted to $6.0 \times 10^{13} \mathrm{QDs} \mathrm{mL}^{-1}(100 \mathrm{nM})$ with $\mathrm{CHCl}_{3}$ was used. The absorbance spectrum for such commercial CdSe QDs provided a band centered at $560 \mathrm{~nm}$, which could be employed for the estimation of their size following Peng's method [23], $3.30 \pm 0.15 \mathrm{~nm}$. A size of $3.34 \pm 0.07 \mathrm{~nm}$ was found with the electrochemical method proposed. The high similarity of the results obtained by both methods, validates the here proposed electrochemical method.

In brief, the electrochemical method developed in this work presents some advantages or disadvantages as compared to other alternative methods already described for QDs characterization. The here-proposed method requires sample acid attack and does not provide simultaneously size and concentration information. However it is fast, simple, of relatively low cost and could be used for routine control of QDs synthesis.

Alternatively, the use of spectrophotometry measurements and Peng's calculations is also a fast and simple approach that does not require sample treatments. However, it has several important limitations, including that it cannot be directly applied for QDs of a different core nature. Moreover, results greatly depend on spectrochemical interferences (e.g. any concomitant species eventually absorbing UV-light in the same spectral region would interfere in the final result).

Transmission electron microscopy (TEM) provides reliable information about the QDs particle size. However, it is not useful for routine control of samples containing QDs and it could suffer from aggregation problems during sample pretreatment particularly for high concentrated samples. In addition it should be considered the reduced availability of such instrumentation.

ICP-MS in combination with TEM or XRD has been also used for characterization of the QDs (size and concentration). The technique is highly sensitive for metal determination and it is highly robust suffering of low matrix interferences. However, acquisition and running costs are high. Moreover, it requires digestion of the QDs for reliable concentration determinations, as it occurs in the method proposed herein.

Other described methodologies provide nanoparticle concentrations in terms of mass of solid per volume (i.e. obtained after drying a given volume of colloidal samples). However, as stated in the introduction, such information is very limited and usefulness in bioconjugation or toxicological studies. Instead of that, expression of the nanoparticle concentration in terms of number of nanoparticles in a given sample volume, as carried out in the approach here proposed, is strongly needed for a proper study of toxicological or bioanalytical uses of QDs.

\section{Conclusions}

A simple, inexpensive and fast method has been developed for the determination of the nanoparticle concentration of Cd-based
QDs and for their size characterization. The simplicity of the methodology and the use of screen-printed electrodes allow for the characterization of the metal nanoparticle by a non-specialist technician. Fast electrochemical characterization of QDs in organic medium could be used as screening at an early stage of the synthesis before introducing the shell and subsequent functionalizations. Therefore, the information provided by the electrochemical methodology could complement the more established techniques used in routine analysis during QDs synthesis for quality control of the nanoparticles. The sensitivity provided by the electrochemical technique (anodic stripping voltammetry) results in the detection of very low concentrations of QDs, and the possibility to identify a wide range of sizes.

It is worth mentioning that the developed methodology could be applied to other types of QDs with an electroactive metal in the core, extending the use of this technique to a larger scope.

\section{Acknowledgements}

This work has been supported by MICINN-12-CTQ201124560 and MINECO-13-CTQ2013-49032-C2-1-R projects from the Spanish Ministery of Economy and Competitiveness (MEC). Daniel Martín-Yerga thanks the MEC for the award of a FPI grant (BES-2011-054408). M.M.M. and D.B.R. acknowledge the Ph.D. grants (BP12-046 and BP14-137, respectively) from Principado de Asturias (Spain).

\section{References}

[1] A. Ekimov, A. Onushchenko, Quantum size effect in three-dimensional microscopic semiconductor crystals, ZhETF Pis Ma Redaktsiiu. 34 (1981) 363-366.

[2] A.P. Alivisatos, Semiconductor Clusters Nanocrystals, and Quantum Dots, Science (80-.) 271 (1996) 933-937.

[3] H. Weller, Colloidal Semiconductor Q-Particles: Chemistry in the Transition Region Between Solid State and Molecules, Angew. Chem., Int. Ed. 32 (1993) 41-53.

[4] B.O. Dabbousi, J. Rodriguez-Viejo, F.V. Mikulec, J.R. Heine, H. Mattoussi, R. Ober, et al., (CdSe)ZnS Core-Shell Quantum Dots: Synthesis and Characterization of a Size Series of Highly Luminescent Nanocrystallites, J. Phys. Chem. B. 101 (1997) 9463-9475.

[5] A.R. Montoro Bustos, J.R. Encinar, M.T. Fernández-Argüelles, J.M. CostaFernández, A. Sanz-Medel, Elemental mass spectrometry: a powerful tool for an accurate characterisation at elemental level of quantum dots, Chem. Commun. (Cambridge, U. K.) (2009) 3107-3109.

[6] J. Drbohlavova, V. Adam, R. Kizek, J. Hubalek, Quantum dots - characterization, preparation and usage in biological systems, Int. J. Mol. Sci. 10 (2009) 656-673.

[7] L. Trapiella-Alfonso, A.R. Montoro Bustos, J.R. Encinar, J.M. Costa-Fernández, R. Pereiro, A. Sanz-Medel, New integrated elemental and molecular strategies as a diagnostic tool for the quality of water soluble quantum dots and their bioconjugates, Nanoscale. 3 (2011) 954-957.

[8] S.K. Haram, B.M. Quinn, A.J. Bard, Electrochemistry of CdS nanoparticles: a correlation between optical and electrochemical band gaps, J. Am. Chem. Soc. 123 (2001) 8860-8861.

[9] M. Amelia, S. Impellizzeri, S. Monaco, I. Yildiz, S. Silvi, F.M. Raymo, et al., Structural and size effects on the spectroscopic and redox properties of CdSe nanocrystals in solution: the role of defect states, Chemphyschem. 12 (2011) 2280-2288.

[10] S.K. Haram, A. Kshirsagar, Y.D. Gujarathi, P.P. Ingole, O. a. Nene, G.B. Markad, et al., Quantum Confinement in CdTe Quantum Dots: Investigation through Cyclic Voltammetry Supported by Density Functional Theory (DFT), J. Phys. Chem. C. 115 (2011) 6243-6249.

[11] Y. Bae, N. Myung, A.J. Bard, Electrochemistry and electrogenerated chemiluminescence of CdTe nanoparticles, Nano Lett. 4 (2004) 1153-1161.

[12] A.J. Bard, Z. Ding, N. Myung, Electrochemistry and electrogenerated chemiluminescence of semiconductor nanocrystals in solutions and in films, in: Struct. Bond. (2005) 1-57.

[13] M. Amelia, C. Lincheneau, S. Silvi, A. Credi, Electrochemical properties of CdSe and CdTe quantum dots, Chem. Soc. Rev. 41 (2012) 5728-5743.

[14] H. Huang, J.-J. Zhu, The electrochemical applications of quantum dots, Analyst 138 (2013) 5855-5865.

[15] A. Merkoçi, L.H. Marcolino-Junior, S. Marín, O. Fatibello-Filho, S. Alegret, Detection of cadmium sulphide nanoparticles by using screen-printed electrodes and a handheld device, Nanotechnology 18 (2007) 35502.

[16] P. Sobrova, M. Ryvolova, J. Hubalek, V. Adam, R. Kizek, Voltammetry as a Tool for Characterization of CdTe Quantum Dots, Int. J. Mol. Sci. 14 (2013) 13497-13510. 
[17] D. Hynek, M., Zurek, P., Babula, V., Adam, R. Kizek, Monitoring of the Surface Modification of Nanoparticles by Electrochemical Measurements Using Scanning Electrochemical Microscope, in: Mod. Electrochem. Methods Nano, Surf. Corros. Sci. 2014: pp. 139-170.

[18] R. Gill, M. Zayats, I. Willner, Semiconductor quantum dots for bioanalysis, Angew. Chem., Int. Ed. 47 (2008) 7602-7625.

[19] Y.L. Liu, J. Zhu, P. Kong, B. Yang Liu, A Quantum Dots-Based Electrochemical Assay Towards the Sensitive Detection of Tumor Cells, Electrochem. Commun. 33 (2013) 59-62.

[20] F. Lisdat, D. Schäfer, A. Kapp, Quantum dots on electrodes-new tools for bioelectroanalysis, Anal. Bioanal. Chem. 405 (2013) 3739-3752.

[21] Z.A. Peng, X. Peng, Nearly Monodisperse and Shape-Controlled CdSe Nanocrystals via Alternative Routes: Nucleation and Growth, J. Am. Chem. Soc. 124 (2002) 3343-3353.

[22] M.T. Fernández-Argüelles, A. Yakovlev, R.A. Sperling, C. Luccardini, S. Gaillard, A. Sanz-Medel, et al., Synthesis and characterization of polymer-coated quantum dots with integrated acceptor dyes as FRET-based nanoprobes, Nano Lett. 7 (2007) 2613-2617.

[23] W.W. Yu, L. Qu, W.X. Guo Peng, Experimental Determination of the Extinction Coefficient of CdTe, CdSe, and CdS Nanocrystals, Chem. Mater. 15 (2003) 2854-2860.

[24] W. Guo, J.J. Li, Y.A. Wang, X. Peng, Luminescent CdSe/CdS core/shell nanocrystals in dendron boxes: Superior chemical, photochemical and thermal stability, J. Am. Chem. Soc. 125 (2003) 3901-3909.
[25] L. Wang, D.K. Nagesha, S. Selvarasah, M.R. Dokmeci, R.L. Carrier, Toxicity of CdSe Nanoparticles in Caco-2Cell Cultures, J. Nanobiotechnol. 6 (2008) 11.

[26] R.T. Kachoosangi, C.E. Banks, X. Ji, R.G. Compton, Electroanalytica determination of cadmium(II) and lead(II) using an in-situ bismuth film modified edge plane pyrolytic graphite electrode, Anal. Sci. 23 (2007) 283-289.

[27] N. Serrano, J.M. Díaz-Cruz, C. Ariño, M. Esteban, Stripping analysis of heavy metals in tap water using the bismuth film electrode, Anal. Bioanal. Chem. 396 (2010) 1365-1369.

[28] N. Serrano, J.M. Díaz-Cruz, C. Ariño, M. Esteban, Ex situ Deposited Bismuth Film on Screen-Printed Carbon Electrode: A Disposable Device for Stripping Voltammetry of Heavy Metal Ions, Electroanalysis 22 (2010) 1460-1467.

[29] N. Serrano, A. Alberich, J.M. Díaz-Cruz, C. Ariño, M. Esteban, Coating methods, modifiers and applications of bismuth screen-printed electrodes, TrAC Trends Anal. Chem. 46 (2013) 15-29.

[30] D. Martín-Yerga, M.B. González-García, A. Costa-García, Biosensor array based on the in situ detection of quantum dots as electrochemical label, Sens. Actuators B. 182 (2013) 184-189.

[31] D. Martín-Yerga, M.B. González-García, A. Costa-García, Electrochemical immunosensor for anti-tissue transglutaminase antibodies based on the in situ detection of quantum dots, Talanta 130 (2014) 598-602.

[32] J. Wang, G. Liu, A. Merkoçi, Electrochemical coding technology for simultaneous detection of multiple DNA targets, J. Am. Chem. Soc. 125 (2003) 3214-3215. 